\title{
Alcohol and Tobacco use While Breastfeeding and Risk of Autism Spectrum Disorder or Attention Deficit/Hyperactivity Disorder
}

\author{
Louisa Gibson $^{1}$ (D) . Melanie Porter ${ }^{1}$ (D) \\ Accepted: 14 April 2021 / Published online: 24 April 2021 \\ (c) The Author(s), under exclusive licence to Springer Science+Business Media, LLC, part of Springer Nature 2021
}

\begin{abstract}
Research has linked prenatal alcohol and tobacco use with Attention Deficit Hyperactivity Disorder (ADHD), and variably with Autism Spectrum Disorder (ASD). Lactational use has been scantly considered. This study examined whether it may alter ADHD or ASD risk. Participants were 5107 infants recruited in 2004 and assessed longitudinally for the Growing Up in Australia Study. Logistic regression did not find any associations between maternal alcohol and tobacco use while breastfeeding and ADHD or ASD diagnosis at ages 6-7 or 10-11 years. Alcohol and tobacco use during lactation may not increase ADHD or ASD risk. Abstaining from alcohol and tobacco, however, may still be the safest option. Analyses were limited by lack of alcohol timing and retrospective variables that future research should address.
\end{abstract}

Keywords Autism spectrum disorder $\cdot$ Attention deficit/hyperactivity disorder $\cdot$ Breastfeeding $\cdot$ Alcohol $\cdot$ Tobacco

\section{Introduction}

Prenatal alcohol and tobacco exposure are associated with Attention Deficit/Hyperactivity Disorder (ADHD) (Dong et al., 2018; Huang et al., 2018; Wetherill et al., 2018). While findings are variable, prenatal tobacco exposure (Gardener et al., 2009; Jung et al., 2017; Rosen et al., 2015; Tang et al., 2015), but not alcohol (Gallagher et al., 2018; Singer et al., 2017), is also a potential risk factor for Autism Spectrum Disorder (ASD). This may be related to brain development, since prenatal alcohol and nicotine are associated with negative brain changes (Bublitz \& Stroud, 2012; Ekblad et al., 2015; Lebel et al., 2011; Paolozza et al., 2014). Breastfeeding alcohol has also been associated with decreased abstract reasoning (Gibson \& Porter, 2018) and academic outcomes (Gibson \& Porter, 2020a). Given these associations, it is possible that alcohol or tobacco intake during lactation may also be related to ADHD or ASD.

Louisa Gibson

louisa.gibson@hdr.mq.edu.au

Melanie Porter

melanie.porter@mq.edu.au

1 Department of Psychology, Faculty of Medicine, Health and Human Sciences, Macquarie University, Balaclava Road, North Ryde, Sydney, NSW 2109, Australia
Prenatal tobacco is related to decreased brain volume, and alterations to information and auditory processing (Bublitz \& Stroud, 2012; Ekblad et al., 2015). In particular, reductions in frontal lobe volume (Bublitz \& Stroud, 2012; Ekblad et al., 2015) may underlie difficulties with attention (Cornelius et al., 2011; Noland et al., 2005), impulsivity (Cornelius et al., 2011), and drug use (Wilens et al., 2011). These changes, combined with genes that are associated with both ADHD and smoking (Barkley et al., 1019), could partly explain associations between maternal smoking and ADHD. While many children with ADHD have not had tobacco exposure (Zhu et al., 2014), meta-analyses have identified increased ADHD risk in children whose mothers smoked while pregnant (Dong et al., 2018; Huang et al., 2018). This relationship may be dose-dependent, since heavier smoking has been associated with greatest risk (Huang et al., 2018). Results regarding prenatal tobacco smoking and ASD are mixed (Gardener et al., 2009; Jung et al., 2017; Rosen et al., 2015; Tang et al., 2015). While several meta-analyses (Gardener et al., 2009; Rosen et al., 2015; Tang et al., 2015) have not identified a relationship, Jung et al. (2017) found that prenatal tobacco smoking was geographically associated with increased ASD risk.

As with tobacco, prenatal alcohol exposure is associated with reduced brain volume (Lebel et al., 2011), and attention and impulsivity difficulties (Paolozza et al., 2014). Dosedependence is also suggested, since children with prenatal 
alcohol exposure and Fetal Alcohol Spectrum Disorder have greater attention difficulties than children with prenatal alcohol exposure alone (Paolozza et al., 2014). The same ADHD and smoking genes have also been associated with alcohol use (Barkley et al., 1019). Although many children with ADHD have not had alcohol exposure (Gustavson et al., 2017), a meta-analysis found that children of mothers who drank during pregnancy had greater risk of developing ADHD (Wetherill et al., 2018). The evidence regarding alcohol and ASD is limited, but does not support an association between prenatal alcohol and ASD (Gallagher et al., 2018; Singer et al., 2017). Mothers of children with ASD were less likely to have consumed alcohol prenatally (Singer et al., 2017), and no increased ASD risk following prenatal alcohol was observed (Gallagher et al., 2018).

Although the teratogenic effects are well researched, assessments of alcohol or tobacco use during lactation are limited. Both alcohol (Kesäniemi, 1974) and nicotine (Luck $\&$ Nau, 1984) pass quickly to breastmilk, and may negatively impact brain development (Borges \& Lewis, 1982; Climent et al., 2002; Gonzalez-Burgos et al., 2006; Hekmatpanah et al., 1994; Museridze et al., 2008; Zhu et al., 1996). The concentration of alcohol in breastmilk is similar to maternal blood alcohol concentration (BAC) (Kesäniemi, 1974). "Pumping and dumping" breastmilk may therefore be ineffective at reducing breastmilk alcohol (Lawton, 1985). Nicotine concentration in breastmilk may be higher than maternal BAC (Luck \& Nau, 1984). Both alcohol and nicotine reduce milk production (Napierala et al., 2016; Mennella et al., 2005; Giglia \& Binns, 2006), and alcohol can alter infant sleeping patterns (Haastrup et al., 2014; Mennella, 2001). Nicotine is associated with changes in breastmilk composition and taste (Napierala et al., 2016), which may further impact infant feeding and nutrition.

Dose-dependent reductions in abstract reasoning (Gibson \& Porter, 2018), academic achievement (Gibson \& Porter, 2020a), but not developmental health (Gibson \& Porter, 2020b) have been observed following maternal consumption of alcohol while breastfeeding. This suggests that cognitive and academic outcomes may be impacted by maternal intake of alcohol during lactation. It has also been observed that children from language backgrounds other than English match or outperform English-language counterparts on Australian academic tests (Gibson \& Porter, 2021). While causality is unlikely, it is noteworthy that these children breastfed for longer, and their mothers consumed less alcohol or had less risky alcohol consumption patterns while pregnant and breastfeeding (Gibson \& Porter, 2021).

Prenatal tobacco and alcohol have been identified as potential risk factors for ADHD (Dong et al., 2018; Huang et al., 2018; Wetherill et al., 2018) and ASD (Gallagher et al., 2018; Gardener et al., 2009; Jung et al., 2017; Rosen et al., 2015; Singer et al., 2017; Tang et al., 2015). Additionally, alcohol consumed while breastfeeding has been associated with negative cognitive and academic outcomes in children (Gibson \& Porter, 2018, 2020a). Given these associations, the current study aimed to assess whether tobacco or alcohol intake during lactation may be separately associated with increased ADHD or ASD risk. It was hypothesised that alcohol and tobacco intake during breastfeeding would each be independently dose-dependently related to increased ADHD and ASD risk in children.

\section{Method}

\section{Study Cohort}

Data was sourced from Growing Up in Australia: The Longitudinal Study of Australian Children (LSAC) (Growing Up in Australia n.d.). Analyses comprised 5107 infants and caregivers from LSAC recruited in 2004 (average age $=9$ months, range $=4-19$ months). Participants were assessed in data waves every two years (Australian Institute of Family Studies, 2015). Wave 1 represents study entry. Only waves 4 (age 6-7 years) and 6 (age 10-11 years) outcomes were analysed for comparison to prior studies (Gibson \& Porter, 2018, 2020a, 2020b). Further details are available in LSAC Technical Paper No 1 (Soloff et al., 2005), from the LSAC website https://growingupinaustralia.gov.au/ (including dietary information), and from previous publications (Gibson \& Porter, 2018, 2020a, 2020b).

\section{Breastfeeding}

Children were grouped into babies who were breastfeeding at Wave 1 (sample group 1), and babies who had been breastfed at any time (babies who were actively breastfeeding at Wave 1 combined with babies who had been breastfed in the past, but had stopped by study entry) (sample group 2) (Fig. 1). Questionnaires asked whether babies were currently breastfed or previously breastfed. Breastfeeding was not defined any further, and the questionnaire did not specify whether feeding of expressed milk should be defined as breastfeeding. Further details, including specific wording of questions, have been previously described (Gibson \& Porter, 2018, 2020a, 2020b).

\section{Predictor Variables}

Variables have been described previously (Gibson \& Porter, 2018, 2020a, 2020b). Briefly, tobacco smoking was recorded as the number of cigarettes mothers smoked on average per day at Wave 1 , and retrospectively during pregnancy. Alcohol was measured using a modified version of the alcohol use disorders identification test 
Fig. 1 Graphical representation of study samples. Numbers given are after multiple imputation

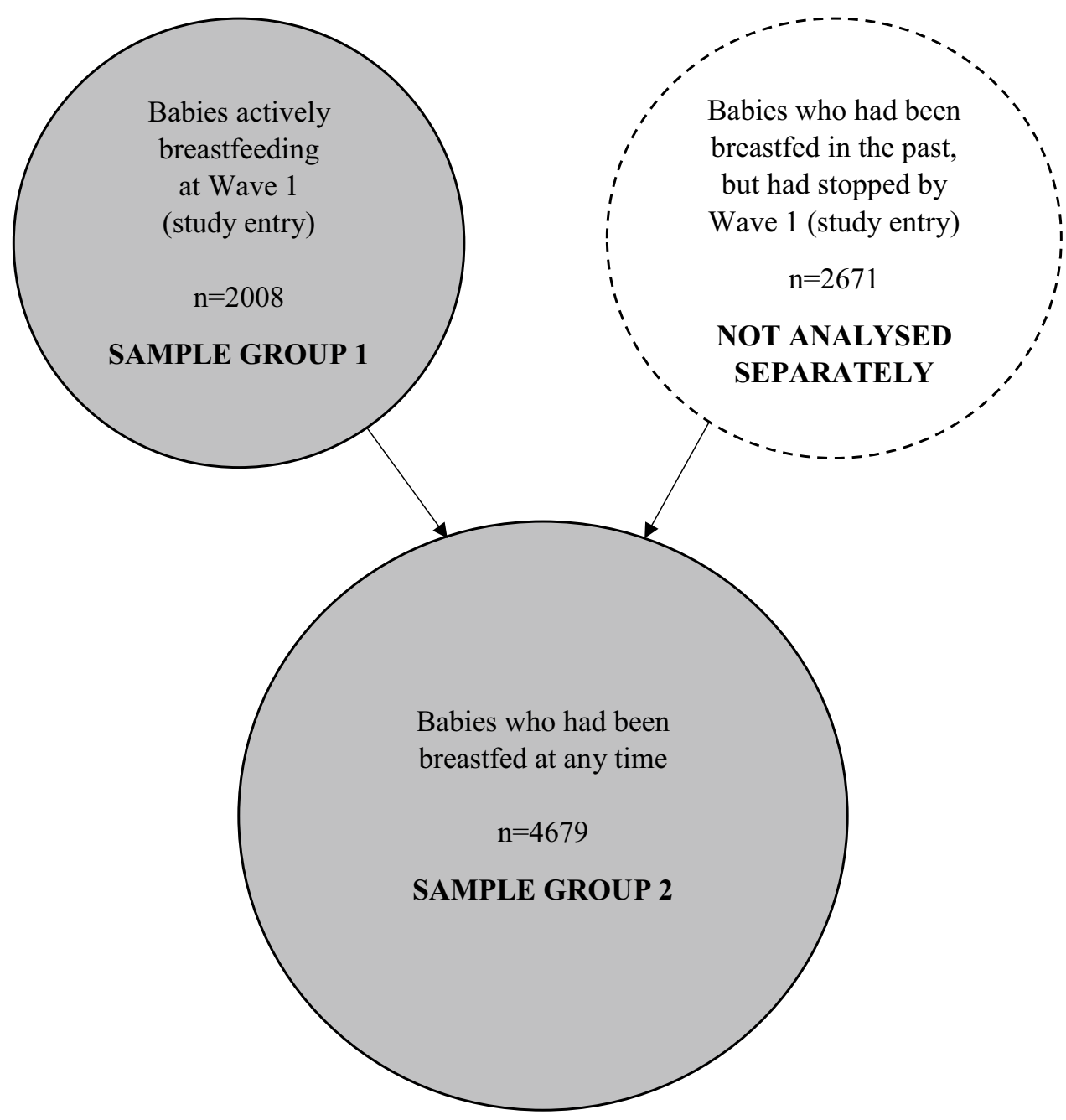

(AUDIT) Alcohol Consumption Questions (AUDIT-C) (Babor et al., 2001; Bush et al., 1998) at Wave 1. Higher scores indicated greater or riskier alcohol consumption. These self-report questions were the only methods employed for measuring tobacco and alcohol use. A visual chart explaining the concept of a standard drink was provided, but the type of alcohol consumed was not asked. The modified AUDIT-C questions were as below.

(1) Mother's frequency of drinking alcohol?

Never; Not in the last year; Monthly or less; 2 to 3 times per month; Once a week; 2 to 3 times a week; 4 to 6 times a week; Every day.

(2) Mother's average number of drinks when drinking? $0 ; 1$ or $2 ; 3$ or $4 ; 5$ or $6 ; 7$ to $10 ; 11$ or more.

(3) Mother's frequency of drinking $\geq 5$ drinks in one sitting?

Not in the last year; Monthly or less; 2 or 3 times a month; Once a week; 2 to 3 times a week; 4 to 6 times a week; Every day.
Pregnancy alcohol consumption was recorded retrospectively as the number of days per week mothers drank alcohol each trimester and the average quantity they consumed on each occasion as below. No other method of measurement was utilised.

(1) Mother's trimester 1 days per week drinking alcohol? 0 or occasional; $1 ; 2 ; 3 ; 4 ; 5 ; 6 ; 7$.

(2) Mother's trimester 2 days per week drinking alcohol? 0 or occasional; $1 ; 2 ; 3 ; 4 ; 5 ; 6 ; 7$.

(3) Mother's trimester 3 days per week drinking alcohol? 0 or occasional; $1 ; 2 ; 3 ; 4 ; 5 ; 6 ; 7$.

(4) Average number of drinks on drinking days? 0 or none; 1 or $2 ; 3$ or $4 ; 5$ or $6 ; 7$ to $10 ; 11$ or more.

\section{Outcome Variables}

Outcome variables were a diagnosis of ADHD or ASD at Waves 4 and 6 as reported by caregivers. No separate assessment was conducted, and dual diagnosis was not 
considered. While caregivers were not questioned about the diagnostic process, the Diagnostic and Statistical Manual of Mental Disorders, fourth edition (American Psychiatric Association, 1994) was the standard diagnostic manual among mental health professionals in Australia at the time. The questions to caregivers regarding ADHD and ASD diagnosis were worded as follows, with the option to answer yes or no:

(1) Does child have any of these ongoing conditions? Autism, Aspergers [sic], or other autism spectrum [sic]?

(2) Does child have any of these ongoing conditions? ADD[sic]/ADHD?

\section{Control Variables}

Control variables have been described previously (Gibson \& Porter, 2018, 2020a, 2020b). Briefly, they included sex, child age, maternal age, combined family income, maternal education, birthweight, and breastfeeding duration, since these have all been associated with cognitive or academic outcomes in children (Aarnoudse-Moens et al., 2009; Bernard et al., 2017; Leigh \& Gong, 2010; Semrud-Clikeman, 2005; Stoet \& Geary, 2013; Tong et al., 2007; Van Heugten et al., 2006; Verhaeghen \& Salthouse, 1997). As described previously (Gibson \& Porter, 2020a), in analyses of babies who had been breastfed at any time, breastfeeding status (current or prior) was added as a control variable to account for non-contemporaneous measurement of maternal modified AUDIT-C scores and maternal smoking in infants who had ceased breastfeeding by study entry.

\section{Statistical Analyses}

Data was analysed using IBM SPSS version 24. Missing data was imputed using multiple imputation (MI) according to previous methods (Gibson \& Porter, 2018). Twenty-eight imputations were used since the highest proportion of missing data for any variable was $28 \%$ (Gibson \& Porter, 2018, 2020a, 2020b). Matching the imputation number to missing data percentage when missing data is $<50 \%$, increases efficiency and replicability of data (von Hippel, 2016).

Multivariable logistic regression analyses were performed including all predictor and control variables separately for each outcome variable for consistency with prior research (Gibson \& Porter, 2018, 2020a, 2020b). The Benjamini-Hochberg procedure (Benjamini \& Hochberg, 1995) was used to correct for Type I error ( $\alpha=0 \cdot 05,2$-tailed). The variance explained by the model was reported using the Nagelkerke pseudo R Square.

\section{Results}

\section{Descriptive Statistics (Prior to MI)}

Descriptive statistics have been previously reported (Gibson \& Porter, 2018, 2020a, 2020b).

\section{Power Analyses}

Only data from biological mothers and their children was included. With 14 independent variables, and a pooled sample size of 2008 infants (after MI) who were breastfeeding at study entry, $>99 \%$ power was achieved $(d=0 \cdot 2$, $\alpha=0.05)$. With a sample of 4679 babies who had breastfed at any time, $>99 \%$ power was also achieved $(d=0 \cdot 2$, $\alpha=0.05$ ) with 15 independent variables (Faul et al., 2007, 2009).

\section{Missing Data}

Little's Missing Completely at Random (MCAR) test found that data was not MCAR, $\chi 2=3617.52, \mathrm{df}=1889$, $\mathrm{p}={ }^{<} 0.0001$. Data may be missing at random and suitable for MI (Sterne et al., 2009), given that poorly educated parents were more likely to drop out of the study (Baxter, 2013).

\section{Babies Breastfeeding at Wave 1: ADHD}

For Wave 4, the model explained $4-17 \%$ of variance across imputations. For Wave 6, the model explained 3-6\% of variance across imputations. No variables were associated with a greater or lesser ADHD risk (Tables 1,2).

\section{Babies Breastfeeding at Wave 1: ASD}

For Wave 4, the model explained 4-7\% of variance across imputations. For Wave 6, the model explained $2-7 \%$ of variance across imputations. No variables were associated with a greater or lesser ASD risk (Tables 3, 4).

\section{Babies Breastfed at Any Time: ADHD}

For Wave 4, the model explained 3-5\% of variance across imputations. For Wave 6 , the model explained $2-4 \%$ of variance across imputations. No variables were associated with a greater or lesser ADHD risk (Tables 5, 6).

\section{Babies Breastfed at Any Time: ASD}

For Wave 4, the model explained $1-2 \%$ of variance across imputations. For Wave 6 , the model explained $1-2 \%$ of 
Table 1 Wave 4 attention deficit/hyperactivity disorder (babies breastfeeding at study entry)

\begin{tabular}{|c|c|c|c|c|c|c|}
\hline Variable & $\mathrm{B}$ & SE & Odds Ratio & $95 \% \mathrm{CI}$ & $\mathrm{p}$ value & $\begin{array}{l}\text { Adjusted } \\
\text { p value* }\end{array}$ \\
\hline Mother's modified AUDIT-C score wave 1 & 0.19 & 0.12 & 1.21 & $0.96-1.53$ & 0.10 & 0.95 \\
\hline Mother's level of education & -0.26 & 0.18 & 0.77 & $0.54-1.09$ & 0.14 & 0.95 \\
\hline Child's birth weight (grams) & 0.00 & 0.00 & 1.00 & $1.00-1.00$ & 0.31 & 0.95 \\
\hline Mother's average daily cigarettes wave 1 & -0.12 & 0.18 & 0.89 & $0.62-1.26$ & 0.50 & 0.95 \\
\hline Child's sex & -0.31 & 0.51 & 0.73 & $0.27-1.99$ & 0.54 & 0.95 \\
\hline Pregnancy: average number of drinks & -0.34 & 0.65 & 0.71 & $0.20-2.58$ & 0.61 & 0.95 \\
\hline Pregnancy: 3rd trimester days per week drank alcohol & -0.51 & 1.00 & 0.60 & $0.08-4.27$ & 0.61 & 0.95 \\
\hline Combined family income $\mathrm{e}^{\#}$ & -0.05 & 0.13 & 0.95 & $0.74-1.22$ & 0.69 & 0.95 \\
\hline Mother's age wave 1 & -0.02 & 0.05 & 0.98 & $0.89-1.09$ & 0.75 & 0.95 \\
\hline Breastfeeding duration (days) & 0.00 & 0.00 & 1.00 & $1.00-1.00$ & 0.79 & 0.95 \\
\hline Child's age (months) & 0.19 & 0.86 & 1.21 & $0.23-6.52$ & 0.82 & 0.95 \\
\hline Pregnancy: 2nd trimester days per week drank alcohol & 0.17 & 0.99 & 1.18 & $0.17-8.28$ & 0.86 & 0.95 \\
\hline Average daily cigarettes while pregnant & -0.03 & 0.25 & 0.97 & $0.60-1.58$ & 0.91 & 0.95 \\
\hline Pregnancy: 1st trimester days per week drank alcohol & 0.05 & 0.69 & 1.05 & $0.27-4.05$ & 0.95 & 0.95 \\
\hline Constant & -5.90 & 6.47 & 0.00 & $0.00-886.65$ & 0.36 & N/A \\
\hline
\end{tabular}

AUDIT-C alcohol use disorders identification test alcohol consumption questions, $S E$ standard error, $C I$ confidence interval

${ }^{\#}$ Higher scores indicate lower income

*Benjamini-Hochberg method

Table 2 Wave 6 attention deficit/hyperactivity disorder (babies breastfeeding at study entry)

\begin{tabular}{|c|c|c|c|c|c|c|}
\hline Variable & B & SE & Odds Ratio & $95 \%$ CI & $\mathrm{p}$ value & $\begin{array}{l}\text { Adjusted } \\
\text { p value* }\end{array}$ \\
\hline Pregnancy: 3rd trimester days per week drank alcohol & 0.55 & 0.21 & 1.73 & $1.14-2.63$ & 0.01 & 0.08 \\
\hline Pregnancy: average number of drinks & 0.68 & 0.27 & 1.97 & $1.16-3.34$ & 0.01 & 0.08 \\
\hline Pregnancy: 2nd trimester days per week drank alcohol & -0.72 & 0.38 & 0.49 & $0.23-1.02$ & 0.06 & 0.27 \\
\hline Child's birth weight (grams) & 0.00 & 0.00 & 1.00 & $1.00-1.00$ & 0.12 & 0.40 \\
\hline Mother's modified AUDIT-C score wave 1 & -0.09 & 0.08 & 0.92 & $0.79-1.07$ & 0.26 & 0.73 \\
\hline Breastfeeding duration (days) & 0.00 & 0.00 & 1.00 & $1.00-1.00$ & 0.35 & 0.82 \\
\hline Mother's age wave 1 & -0.02 & 0.03 & 0.98 & $0.93-1.04$ & 0.48 & 0.93 \\
\hline Average daily cigarettes while pregnant & -0.07 & 0.11 & 0.93 & $0.75-1.16$ & 0.53 & 0.93 \\
\hline Child's age (months) & -0.19 & 0.40 & 0.82 & $0.38-1.80$ & 0.63 & 0.95 \\
\hline Mother's level of education & 0.03 & 0.10 & 1.04 & $0.86-1.25$ & 0.72 & 0.95 \\
\hline Pregnancy: 1st trimester days per week drank alcohol & -0.11 & 0.33 & 0.90 & $0.47-1.73$ & 0.75 & 0.95 \\
\hline Combined family income $\mathrm{e}^{\#}$ & -0.01 & 0.06 & 0.99 & $0.88-1.11$ & 0.82 & 0.95 \\
\hline Child's sex & -0.03 & 0.28 & 0.97 & $0.57-1.66$ & 0.91 & 0.98 \\
\hline Mother's average daily cigarettes wave 1 & 0.00 & 0.07 & 1.00 & $0.87-1.15$ & 0.99 & 0.99 \\
\hline Constant & -2.22 & 4.54 & 0.11 & $0.00-802.50$ & 0.63 & N/A \\
\hline
\end{tabular}

AUDIT-C alcohol use disorders identification test alcohol consumption questions, $S E$ standard error, $C I$ confidence interval

${ }^{\#}$ Higher scores indicate lower income

*Benjamini-Hochberg method

variance across imputations. No variables were associated with a greater or lesser ASD risk (Tables 7, 8).

\section{Discussion}

Maternal alcohol and tobacco intake while breastfeeding did not impact ADHD or ASD risk in either sample group 
Table 3 Wave 4 autistic spectrum disorder (babies breastfeeding at study entry)

\begin{tabular}{|c|c|c|c|c|c|c|}
\hline Variable & $\mathrm{B}$ & SE & Odds Ratio & $95 \% \mathrm{CI}$ & $\mathrm{p}$ value & $\begin{array}{l}\text { Adjusted } \\
\text { p value* }\end{array}$ \\
\hline Breastfeeding duration (days) & 0.00 & 0.00 & 1.00 & $1.00-1.00$ & 0.11 & 0.63 \\
\hline Child's sex & -0.57 & 0.36 & 0.57 & $0.28-1.15$ & 0.12 & 0.63 \\
\hline Pregnancy: Average number of drinks & -0.62 & 0.42 & 0.54 & $0.24-1.22$ & 0.14 & 0.63 \\
\hline Mother's modified AUDIT-C score wave 1 & 0.11 & 0.08 & 1.11 & $0.95-1.31$ & 0.18 & 0.63 \\
\hline Average daily cigarettes while pregnant & 0.09 & 0.09 & 1.10 & $0.92-1.31$ & 0.31 & 0.87 \\
\hline Pregnancy: 1st trimester days per week drank alcohol & -0.32 & 0.42 & 0.72 & $0.32-1.66$ & 0.44 & 0.88 \\
\hline Pregnancy: 2nd trimester days per week drank alcohol & 0.46 & 0.65 & 1.58 & $0.45-5.63$ & 0.48 & 0.88 \\
\hline Mother's level of education & -0.08 & 0.13 & 0.93 & $0.73-1.19$ & 0.55 & 0.88 \\
\hline Pregnancy: 3rd trimester days per week drank alcohol & -0.28 & 0.57 & 0.76 & $0.25-2.33$ & 0.63 & 0.88 \\
\hline Child's birth weight (grams) & 0.00 & 0.00 & 1.00 & $1.00-1.00$ & 0.68 & 0.88 \\
\hline Mother's age Wave 1 & 0.01 & 0.04 & 1.01 & $0.95-1.09$ & 0.69 & 0.88 \\
\hline Child's age (months) & 0.19 & 0.62 & 1.21 & $0.36-4.05$ & 0.76 & 0.89 \\
\hline Mother's average daily cigarettes wave 1 & -0.01 & 0.07 & 0.99 & $0.86-1.14$ & 0.88 & 0.89 \\
\hline Combined family income ${ }^{\#}$ & 0.01 & 0.08 & 1.01 & $0.87-1.18$ & 0.89 & 0.89 \\
\hline Constant & -4.20 & 4.58 & 0.02 & $0.00-117.59$ & 0.36 & N/A \\
\hline
\end{tabular}

$A U D I T-C$ alcohol use disorders identification test alcohol consumption questions, $S E$ standard error, $C I$ confidence interval

${ }^{\#}$ Higher scores indicate lower income

*Benjamini-Hochberg method

Table 4 Wave 6 autistic spectrum disorder (babies breastfeeding at study entry)

\begin{tabular}{|c|c|c|c|c|c|c|}
\hline Variable & B & SE & Odds Ratio & $95 \% \mathrm{CI}$ & $\mathrm{p}$ value & $\begin{array}{l}\text { Adjusted } \\
\text { p value* }\end{array}$ \\
\hline Child's age (months) & -0.65 & 0.40 & 0.52 & $0.24-1.15$ & 0.11 & 0.56 \\
\hline Pregnancy: 3rd trimester days per week drank alcohol & 0.34 & 0.22 & 1.41 & $0.91-2.18$ & 0.13 & 0.56 \\
\hline Child's sex & -0.34 & 0.27 & 0.71 & $0.42-1.20$ & 0.20 & 0.56 \\
\hline Pregnancy: Average number of drinks & 0.37 & 0.29 & 1.44 & $0.81-2.56$ & 0.21 & 0.56 \\
\hline Child's birth weight (grams) & 0.00 & 0.00 & 1.00 & $1.00-1.00$ & 0.22 & 0.56 \\
\hline Pregnancy: 2nd trimester days per week drank alcohol & -0.42 & 0.36 & 0.66 & $0.33-1.32$ & 0.24 & 0.56 \\
\hline Breastfeeding duration (days) & 0.00 & 0.00 & 1.00 & $1.00-1.00$ & 0.31 & 0.61 \\
\hline Combined family income ${ }^{\#}$ & -0.05 & 0.06 & 0.95 & $0.85-1.07$ & 0.42 & 0.73 \\
\hline Mother's age wave 1 & -0.02 & 0.03 & 0.98 & $0.93-1.04$ & 0.54 & 0.84 \\
\hline Mother's level of education & -0.05 & 0.09 & 0.95 & $0.79-1.15$ & 0.61 & 0.85 \\
\hline Pregnancy: 1st trimester days per week drank alcohol & -0.10 & 0.31 & 0.91 & $0.49-1.67$ & 0.75 & 0.96 \\
\hline Mother's modified AUDIT-C score wave 1 & -0.01 & 0.07 & 0.99 & $0.86-1.15$ & 0.94 & 0.97 \\
\hline Mother's average daily cigarettes wave 1 & 0.00 & 0.06 & 1.00 & $0.88-1.14$ & 0.96 & 0.97 \\
\hline Average daily cigarettes while pregnant & 0.00 & 0.09 & 1.00 & $0.83-1.19$ & 0.97 & 0.97 \\
\hline Constant & 3.78 & 4.56 & 43.65 & $0.01-335,126.29$ & 0.41 & N/A \\
\hline
\end{tabular}

AUDIT- $C$ alcohol use disorders identification test alcohol consumption questions, $S E$ standard error, $C I$ confidence interval

${ }^{\#}$ Higher scores indicate lower income

*Benjamini-Hochberg method

or time point. Likewise, prenatal alcohol and tobacco, child's age, sex and birthweight, mother's age and education, combined family income, breastfeeding duration, and breastfeeding group were not related to ADHD or ASD.
Given these findings, the suggestion that alcohol and tobacco use during lactation may impact these diagnoses was not supported. While there is no directly comparative prior research, prenatal tobacco exposure has previously 
Table 5 Wave 4 attention deficit/hyperactivity disorder (babies who had been breastfed at any time)

\begin{tabular}{|c|c|c|c|c|c|c|}
\hline Variable & $\mathrm{B}$ & SE & Odds Ratio & $95 \% \mathrm{CI}$ & $\mathrm{p}$ value & $\begin{array}{l}\text { Adjusted } \\
\text { p value* }\end{array}$ \\
\hline Child's sex & -0.49 & 0.27 & 0.61 & $0.36-1.05$ & 0.08 & 0.63 \\
\hline Breastfeeding status (currently or previously breastfed) & 0.53 & 0.31 & 1.69 & $0.93-3.07$ & 0.09 & 0.63 \\
\hline Mother's modified AUDIT-C score wave 1 & 0.10 & 0.07 & 1.10 & $0.97-1.25$ & 0.15 & 0.63 \\
\hline Child's birth weight (grams) & 0.00 & 0.00 & 1.00 & $1.00-1.00$ & 0.24 & 0.63 \\
\hline Pregnancy: 1st trimester days per week drank alcohol & -0.42 & 0.38 & 0.66 & $0.31-1.39$ & 0.27 & 0.63 \\
\hline Child's age (months) & 0.50 & 0.45 & 1.64 & $0.68-3.98$ & 0.27 & 0.63 \\
\hline Pregnancy: average number of drinks & -0.36 & 0.35 & 0.70 & $0.35-1.38$ & 0.30 & 0.63 \\
\hline Combined family income ${ }^{\#}$ & 0.05 & 0.06 & 1.05 & $0.94-1.17$ & 0.41 & 0.74 \\
\hline Mother's average daily cigarettes wave 1 & -0.03 & 0.05 & 0.97 & $0.89-1.07$ & 0.53 & 0.74 \\
\hline Mother's age wave 1 & 0.01 & 0.03 & 1.01 & $0.96-1.07$ & 0.64 & 0.74 \\
\hline Average daily cigarettes while pregnant & 0.03 & 0.06 & 1.03 & $0.92-1.14$ & 0.65 & 0.74 \\
\hline Pregnancy: 2nd trimester days per week drank alcohol & 0.22 & 0.48 & 1.25 & $0.48-3.20$ & 0.65 & 0.74 \\
\hline Mother's level of education & -0.04 & 0.10 & 0.96 & $0.80-1.16$ & 0.68 & 0.74 \\
\hline Breastfeeding duration (days) & 0.00 & 0.00 & 1.00 & $1.00-1.00$ & 0.69 & 0.74 \\
\hline Pregnancy: 3rd trimester days per week drank alcohol & 0.05 & 0.44 & 1.05 & $0.45-2.47$ & 0.91 & 0.91 \\
\hline Constant & -9.20 & 3.40 & 0.00 & $0.00-0.08$ & 0.01 & N/A \\
\hline
\end{tabular}

$A U D I T-C$ alcohol use disorders identification test alcohol consumption questions, $S E$ standard error, $C I$ confidence interval

${ }^{\#}$ Higher scores indicate lower income

*Benjamini-Hochberg method

Table 6 Wave 6 attention deficit/hyperactivity disorder (babies who had been breastfed at any time)

\begin{tabular}{|c|c|c|c|c|c|c|}
\hline Variable & B & SE & Odds Ratio & $95 \% \mathrm{CI}$ & $\mathrm{p}$ value & $\begin{array}{l}\text { Adjusted } \\
\text { p value* }\end{array}$ \\
\hline Pregnancy: 3rd trimester days per week drank alcohol & 0.43 & 0.18 & 1.53 & $1.07-2.19$ & 0.02 & 0.22 \\
\hline Pregnancy: Average number of drinks & 0.43 & 0.20 & 1.54 & $1.04-2.26$ & 0.03 & 0.22 \\
\hline Pregnancy: 2nd trimester days per week drank alcohol & -0.53 & 0.29 & 0.59 & $0.33-1.04$ & 0.07 & 0.34 \\
\hline Combined family income $\mathrm{e}^{\#}$ & 0.06 & 0.04 & 1.06 & $0.98-1.15$ & 0.12 & 0.45 \\
\hline Average daily cigarettes while pregnant & -0.07 & 0.06 & 0.93 & $0.83-1.03$ & 0.18 & 0.47 \\
\hline Mother's level of education & 0.09 & 0.07 & 1.09 & $0.96-1.25$ & 0.19 & 0.47 \\
\hline Child's birth weight (grams) & 0.00 & 0.00 & 1.00 & $1.00-1.00$ & 0.24 & 0.50 \\
\hline Breastfeeding status (currently or previously breastfed) & -0.22 & 0.20 & 0.80 & $0.54-1.19$ & 0.27 & 0.50 \\
\hline Pregnancy: 1st trimester days per week drank alcohol & -0.28 & 0.29 & 0.75 & $0.43-1.33$ & 0.33 & 0.54 \\
\hline Breastfeeding duration (days) & 0.00 & 0.00 & 1.00 & $1.00-1.00$ & 0.45 & 0.67 \\
\hline Mother's average daily cigarettes wave 1 & 0.02 & 0.04 & 1.02 & $0.95-1.09$ & 0.55 & 0.75 \\
\hline Child's age (months) & 0.14 & 0.29 & 1.14 & $0.65-2.01$ & 0.64 & 0.80 \\
\hline Child's sex & -0.06 & 0.19 & 0.94 & $0.65-1.36$ & 0.74 & 0.86 \\
\hline Mother's modified AUDIT-C score wave 1 & -0.01 & 0.05 & 0.99 & $0.91-1.09$ & 0.87 & 0.93 \\
\hline Mother's age wave 1 & 0.00 & 0.02 & 1.00 & $0.96-1.04$ & 0.96 & 0.96 \\
\hline Constant & -6.65 & 3.26 & 0.00 & $0.00-0.78$ & 0.04 & N/A \\
\hline
\end{tabular}

AUDIT-C alcohol use disorders identification test alcohol consumption questions, $S E$ standard error, $C I$ confidence interval

${ }^{\#}$ Higher scores indicate lower income

*Benjamini-Hochberg method

been associated with ADHD (Dong et al., 2018; Huang et al., 2018; Wetherill et al., 2018). Although it might be expected that smoking tobacco while breastfeeding may also increase ADHD risk, prior studies have not found breastfeeding tobacco to be related to cognitive, academic or developmental health (Gibson \& Porter, 2018, 2020a, 
Table 7 Wave 4 autistic spectrum disorder (babies who had been breastfed at any time)

\begin{tabular}{|c|c|c|c|c|c|c|}
\hline Variable & B & SE & Odds Ratio & $95 \% \mathrm{CI}$ & $\mathrm{p}$ value & $\begin{array}{l}\text { Adjusted } \\
\text { p value* }\end{array}$ \\
\hline Breastfeeding status (currently or previously breastfed) & 0.32 & 0.23 & 1.38 & $0.88-2.15$ & 0.16 & 0.89 \\
\hline Mother's modified AUDIT-C score wave 1 & 0.06 & 0.05 & 1.06 & $0.97-1.16$ & 0.21 & 0.89 \\
\hline Breastfeeding duration (days) & 0.00 & 0.00 & 1.00 & $1.00-1.00$ & 0.33 & 0.89 \\
\hline Mother's level of education & -0.06 & 0.07 & 0.94 & $0.81-1.09$ & 0.41 & 0.89 \\
\hline Mother's average daily cigarettes wave 1 & -0.02 & 0.03 & 0.98 & $0.92-1.04$ & 0.48 & 0.89 \\
\hline Pregnancy: 3rd trimester days per week drank alcohol & -0.20 & 0.34 & 0.82 & $0.42-1.59$ & 0.56 & 0.89 \\
\hline Child's birth weight (grams) & 0.00 & 0.00 & 1.00 & $1.00-1.00$ & 0.59 & 0.89 \\
\hline Pregnancy: 2nd trimester days per week drank alcohol & 0.18 & 0.38 & 1.19 & $0.56-2.53$ & 0.65 & 0.89 \\
\hline Pregnancy: 1st trimester days per week drank alcohol & -0.11 & 0.24 & 0.90 & $0.56-1.45$ & 0.66 & 0.89 \\
\hline Pregnancy: average number of drinks & 0.09 & 0.21 & 1.09 & $0.73-1.64$ & 0.66 & 0.89 \\
\hline Combined family income ${ }^{\#}$ & 0.02 & 0.05 & 1.02 & $0.93-1.11$ & 0.73 & 0.89 \\
\hline Mother's age wave 1 & 0.01 & 0.02 & 1.01 & $0.97-1.05$ & 0.77 & 0.89 \\
\hline Average daily cigarettes while pregnant & 0.01 & 0.04 & 1.01 & $0.94-1.09$ & 0.79 & 0.89 \\
\hline Child's sex & -0.05 & 0.21 & 0.96 & $0.64-1.43$ & 0.83 & 0.89 \\
\hline Child's age (months) & -0.01 & 0.35 & 0.99 & $0.50-1.97$ & 0.97 & 0.97 \\
\hline Constant & -4.32 & 2.63 & 0.01 & $0.00-2.29$ & 0.10 & N/A \\
\hline
\end{tabular}

AUDIT-C alcohol use disorders identification test alcohol consumption questions, $S E$ standard error, $C I$ confidence interval

\#Higher scores indicate lower income

*Benjamini-Hochberg method

Table 8 Wave 6 autistic spectrum disorder (babies who had been breastfed at any time)

\begin{tabular}{|c|c|c|c|c|c|c|}
\hline Variable & B & SE & Odds Ratio & $95 \% \mathrm{CI}$ & $\mathrm{p}$ value & $\begin{array}{l}\text { Adjusted } \\
\text { p value* }\end{array}$ \\
\hline Child's birth weight (grams) & 0.00 & 0.00 & 1.00 & $1.00-1.00$ & 0.20 & 0.99 \\
\hline Breastfeeding duration (days) & 0.00 & 0.00 & 1.00 & $1.00-1.00$ & 0.21 & 0.99 \\
\hline Pregnancy: 3rd trimester days per week drank alcohol & 0.24 & 0.20 & 1.27 & $0.86-1.88$ & 0.22 & 0.99 \\
\hline Combined family income ${ }^{\#}$ & -0.04 & 0.04 & 0.97 & $0.90-1.04$ & 0.36 & 0.99 \\
\hline Pregnancy: average number of drinks & 0.18 & 0.21 & 1.20 & $0.80-1.79$ & 0.38 & 0.99 \\
\hline Child's sex & -0.15 & 0.18 & 0.86 & $0.61-1.22$ & 0.40 & 0.99 \\
\hline Pregnancy: 1st trimester days per week drank alcohol & -0.09 & 0.19 & 0.91 & $0.63-1.32$ & 0.62 & 0.99 \\
\hline Mother's age wave 1 & -0.01 & 0.02 & 0.99 & $0.96-1.03$ & 0.76 & 0.99 \\
\hline Pregnancy: 2nd trimester days per week drank alcohol & -0.06 & 0.26 & 0.94 & $0.57-1.57$ & 0.82 & 0.99 \\
\hline Mother's average daily cigarettes wave 1 & -0.01 & 0.03 & 0.99 & $0.94-1.05$ & 0.83 & 0.99 \\
\hline Breastfeeding status (currently or previously breastfed) & -0.04 & 0.19 & 0.96 & $0.66-1.40$ & 0.85 & 0.99 \\
\hline Average daily cigarettes while pregnant & 0.00 & 0.04 & 1.00 & $0.93-1.08$ & 0.94 & 0.99 \\
\hline Mother's level of education & 0.00 & 0.06 & 1.00 & $0.89-1.14$ & 0.95 & 0.99 \\
\hline Mother's modified AUDIT-C score wave 1 & 0.00 & 0.05 & 1.00 & $0.91-1.09$ & 0.95 & 0.99 \\
\hline Child's age (months) & 0.01 & 0.27 & 1.01 & $0.59-1.71$ & 0.99 & 0.99 \\
\hline Constant & -3.85 & 3.10 & 0.02 & $0.00-9.32$ & 0.22 & N/A \\
\hline
\end{tabular}

$A U D I T-C$ alcohol use disorders identification test alcohol consumption questions, $S E$ standard error, $C I$ confidence interval

${ }^{\#}$ Higher scores indicate lower income

*Benjamini-Hochberg method 
2020b). Use of tobacco during lactation may therefore be less impactful on infant development than prenatal exposure.

The failure of the current analyses to confirm associations between maternal tobacco smoking and alcohol consumption during pregnancy and ADHD (Dong et al., 2018; Huang et al., 2018; Wetherill et al., 2018), may also reflect weaknesses in the retrospective nature of LSAC pregnancy variables. It has been suggested that these retrospective variables may result in inaccurate measures (Gibson \& Porter, 2018). Additionally, questionnaires were the only methods used for recording all tobacco and alcohol variables. While no relationship between prenatal tobacco and alcohol and ASD was identified, previous studies have been mixed (Gallagher et al., 2018; Gardener et al., 2009; Jung et al., 2017; Rosen et al., 2015; Singer et al., 2017; Tang et al., 2015), and no consensus is available.

Wave 1 smoking measures and AUDIT-C scores were contemporaneous for babies who were actively breastfeeding at Wave 1, but not for infants who had stopped breastfeeding by study entry. Although current or prior breastfeeding status was included to control for this confound, it may still have impacted results.

Given the greater likelihood of mothers with ADHD to consume alcohol and smoke cigarettes (Wilens et al., 2011) and genetic factors (Barkley et al., 1019), the failure to include maternal ASD and ADHD status is a limitation. Other potential control variables including, but not limited to, intrauterine growth restriction and gestational age, may have further improved analyses (Joseph et al., 2017). Furthermore, alcohol and tobacco use were considered individually, and the possibility of additive effects when both were used together was not considered.

The timing of alcohol consumption relative to feeding was also not measured. This, combined with maternal weight, may have allowed calculations of ethanol in breastmilk (Ho et al., 2001). It is therefore not known how much ethanol was available for consumption by the infant (Ho et al., 2001). The amount of ethanol consumed by the infant may influence results if potential negative effects are due solely to neurotoxic effects of alcohol on infants. There are, however, other potential mechanisms by which maternal alcohol consumption while breastfeeding could impact infant development. Use of alcohol during lactation can reduce milk production alter infant's feeding and sleeping patterns (Giglia \& Binns, 2006; Haastrup et al., 2014; Mennella, 2001; Mennella et al., 2005). Alcohol consumption while breastfeeding could therefore indirectly impact infants by reducing their nutritional intake, or altering their sleep.

Future studies should address these confounds. Contemporaneous measures of alcohol and tobacco use may be beneficial, combined with measuring the time between alcohol consumption and infant feeding. Inclusion of maternal
ADHD or ASD as a control variable may also improve analyses.

An additional consideration is the potential impact of the 2019 Coronavirus disease (COVID-19) pandemic on use of alcohol and tobacco. The COVID-19 pandemic and subsequent restrictions on daily living may have impacted the drinking and smoking habits of some individuals. While a substantial number of people have reported drinking and smoking the same or less, approximately $14-31 \%$ and 6-19\% of people have reported an increase in alcohol and tobacco intake respectively (Bommele et al., 2020; Chodkiewicz et al., 2020; Elling et al., 2020; Kim et al., 2020; Neill et al., 2020; Panagiotidis et al., 2020). For women breastfeeding infants during the COVID-19 pandemic, an increase in alcohol or tobacco consumption may result in greater risk of negative outcomes for children (Gibson \& Porter, 2018, 2020a). Had the current study been conducted during the pandemic, a different pattern of alcohol and tobacco use could have impacted results.

Maternal use of alcohol or tobacco were not related to ADHD or ASD at either age or in either sample group. Although there is no direct comparison, this is not consistent with meta-analyses that have found prenatal tobacco smoking and alcohol consumption to be associated with a diagnosis of ADHD (Dong et al., 2018; Huang et al., 2018; Wetherill et al., 2018). The lack of contemporaneous measurement of alcohol and tobacco use in the group of babies who had ceased breastfeeding at study entry was a limitation, as was the lack of maternal ADHD or ASD diagnosis as control variables, and the failure to measure alcohol timing relative to feeding. Future studies should seek to address these confounds. Assessing associations between maternal use of alcohol and tobacco while breastfeeding on a wider range of cognitive, developmental and health outcomes in children may also be beneficial.

Although alcohol and tobacco intake while breastfeeding was not associated with ADHD or ASD, alcohol and tobacco may still be harmful children or interfere with the lactation process if consumed while breastfeeding (Gibson \& Porter, 2018, 2020a; Napierala et al., 2016). The safest option for breastfeeding women may be to abstain from alcohol and tobacco.

Supplementary Information The online version contains supplementary material available at https://doi.org/10.1007/s10803-021-05027-3.

Acknowledgments This paper uses unit record data from Growing Up in Australia, the Longitudinal Study of Australian Children. The study is conducted in partnership between the Department of Social Services (DSS), the Australian Institute of Family Studies (AIFS) and the Australian Bureau of Statistics (ABS). The findings and views reported in this paper are those of the author and should not be attributed to DSS, AIFS or the ABS. Louisa Gibson was also in receipt of a Research Training Program scholarship from the Australian Government Department of Education. 
Funding This study did not receive any funding.

\section{Declarations}

Conflict of interest The authors declare that they have no conflict of interest.

Ethical Approval Ethics approval was obtained from Macquarie University Human Research Ethics Committee (ref no. 5201822862659, 7th April 2017).

\section{References}

Aarnoudse-Moens, C. S. H., Weisglas-Kuperus, N., van Goudoever, J. B., \& Oosterlaan, J. (2009). Meta-analysis of neurobehavioral outcomes in very preterm and/or very low birth weight children. Pediatrics, 124(2), 717-728

American Psychiatric Association (1994). Diagnostic and statistical manual of mental disorders, fourth edition : DSM-IV (Vol. Accessed from https://nla.gov.au/nla.cat-vn1960662). Washington, DC: American Psychiatric Association.

Australian Institute of Family Studies. (2015). Longitudinal Study of Australian Children Data User Guide - November 2015. Australian Institute of Family Studies.

Australian Institute of Family Studies The Longitudinal Study of Australian Children: Frequently asked questions. http://www.growi ngupinaustralia.gov.au/about/faq.html. Accessed 12 October 2016.

Babor, T. F., Higgins-Biddle, J. C., Saunders, J. B., Monteiro, M. G., \& World Health Organization (2001). AUDIT: The alcohol use disorders identification test: Guidelines for use in primary health care, 2nd ed. World Health Organization. https://apps.who.int/ iris/handle/10665/67205

Barkley, R. A., Smith, K. M., \& Fischer, M. (2019). ADHD risk genes involved in dopamine signaling and metabolism are associated with reduced estimated life expectancy at young adult follow-up in hyperactive and control children. [Research Support, N.I.H., Extramural]. American Journal of Medical Genetics. Part B, Neuropsychiatric Genetics: the Official Publication of the International Society of Psychiatric Genetics, 180(3), 175-185

Baxter, J. (2013). Employment characteristics and transitions of mothers in the Longitudinal Study of Australian Children. Melbourne, Australia: Australian Institute of Family Studies.

Benjamini, Y., \& Hochberg, Y. (1995). Controlling the false discovery rate: A practical and powerful approach to multiple testing. Journal of the royal statistical society. Series B (Methodological), 57, 289-300

Bernard, J. Y., Armand, M., Peyre, H., Garcia, C., Forhan, A., De Agostini, M., et al. (2017). Breastfeeding, polyunsaturated fatty acid levels in colostrum and child intelligence quotient at age 5-6 years. Journal of Pediatrics, 183, 43-50.e43

Bommele, J., Hopman, P., Walters, B. H., Geboers, C., Croes, E., Fong, G. T., et al. (2020). The double-edged relationship between COVID-19 stress and smoking: Implications for smoking cessation. Tobacco induced diseases, 18, 63-63. https://doi.org/10. 18332/tid/125580

Borges, S., \& Lewis, P. D. (1982). A study of alcohol effects on the brain during gestation and lactation. Teratology, 25(3), 283-289

Bublitz, M. H., \& Stroud, L. R. (2012). Maternal smoking during pregnancy and offspring brain structure and function: Review and agenda for future research. Nicotine \& Tobacco Research: Official
Journal of the Society for Research on Nicotine and Tobacco, 14(4), 388-397. https://doi.org/10.1093/ntr/ntr191

Bush, K., Kivlahan, D. R., McDonell, M. B., Fihn, S. D., \& Bradley, K. A. (1998). The AUDIT alcohol consumption questions (AUDIT-C): An effective brief screening test for problem drinking. Archives of Internal Medicine, 158(16), 1789-1795

Chodkiewicz, J., Talarowska, M., Miniszewska, J., Nawrocka, N., \& Bilinski, P. (2020). Alcohol consumption reported during the COVID-19 pandemic: The initial stage. International Journal of Environmental Research and Public Health, 17(13), 4677. https:// doi.org/10.3390/ijerph17134677

Climent, E., Pascual, M., Renau-Piqueras, J., \& Guerri, C. (2002). Ethanol exposure enhances cell death in the developing cerebral cortex: Role of brain-derived neurotrophic factor and its signaling pathways. Journal of Neuroscience Research, 68(2), 213-225

Cornelius, M. D., De Genna, N. M., Leech, S. L., Willford, J. A., Goldschmidt, L., \& Day, N. L. (2011). Effects of prenatal cigarette smoke exposure on neurobehavioral outcomes in 10-year-old children of adolescent mothers. Neurotoxicology and Teratology, 33(1), 137-144. https://doi.org/10.1016/j.ntt.2010.08.006

Dong, T., Hu, W., Zhou, X., Lin, H., Lan, L., Hang, B., et al. (2018). Prenatal exposure to maternal smoking during pregnancy and attention-deficit/hyperactivity disorder in offspring: A metaanalysis. Reproductive Toxicology, 76, 63-70. https://doi.org/10. 1016/j.reprotox.2017.12.010

Ekblad, M., Korkeila, J., \& Lehtonen, L. (2015). Smoking during pregnancy affects foetal brain development. Acta Paediatrica, 104(1), 12-18. https://doi.org/10.1111/apa.12791

Elling, J. M., Crutzen, R., Talhout, R., \& de Vries, H. (2020). Tobacco smoking and smoking cessation in times of COVID-19. Tobacco Prevention \& Cessation, 6, 39-39. https://doi.org/10.18332/tpc/ 122753

Faul, F., Erdfelder, E., Buchner, A., \& Lang, A.-G. (2009). Statistical power analyses using $G^{*}$ Power 3.1: Tests for correlation and regression analyses. Behavior Research Methods, 41(4), $1149-1160$

Faul, F., Erdfelder, E., Lang, A.-G., \& Buchner, A. (2007). G* Power 3: A flexible statistical power analysis program for the social, behavioral, and biomedical sciences. Behavior Research Methods, 39(2), 175-191

Gallagher, C., McCarthy, F. P., Ryan, R. M., \& Khashan, A. S. (2018). Maternal alcohol consumption during pregnancy and the risk of autism spectrum disorders in offspring: A retrospective analysis of the millennium cohort study. Journal of Autism \& Developmental Disorders, 48(11), 3773-3782

Gardener, H., Spiegelman, D., \& Buka, S. (2009). Prenatal risk factors for autism: Comprehensivemeta-analysis. The British Journal of Psychiatry, 195(1), 7-14. https://doi.org/10.1192/bjp.bp.108. 051672

Gibson, L., \& Porter, M. (2018). Drinking or smoking while breastfeeding and later cognition in children. Pediatrics. https://doi.org/ $10.1542 /$ peds.2008-1536

Gibson, L., \& Porter, M. (2020a). Drinking or smoking while breastfeeding and later academic outcomes in children. Nutrients. https://doi.org/10.3390/nu12030829

Gibson, L., \& Porter, M. (2020b). Drinking or smoking while breastfeeding and later developmental health outcomes in children. BMC Research Notes. https://doi.org/10.1186/s13104-020-05072-8

Gibson, L., \& Porter, M. (2021). Maternal drinking and smoking. Can it explain the exceptional academic performance of LBOTE children? BMC Research Notes, 14(141). https://doi.org/10.1186/ s13104-021-05544-5

Giglia, R., \& Binns, C. (2006). Alcohol and lactation: A systematic review. Nutrition \& Dietetics, 63(2), 103-116

Gonzalez-Burgos, I., Alejandre-Gomez, M., Olvera-Cortes, M. E., Perez-Vega, M. I., Evans, S., \& Feria-Velasco, A. (2006). 
Prenatal-through-postnatal exposure to moderate levels of ethanol leads to damage on the hippocampal CA1 field of juvenile rats. A stereology and Golgi study. Neuroscience Research, 56(4), 400-408

Growing Up in Australia Publications. Accessed October 06 2017, from.http://www.growingupinaustralia.gov.au/pubs/index.html

Gustavson, K., Ystrom, E., Stoltenberg, C., Susser, E., Suren, P., Magnus, P., et al. (2017). Smoking in pregnancy and child ADHD. Pediatrics, 139(2), e20162509. https://doi.org/10.1542/peds. 2016-2509

Haastrup, M. B., Pottegard, A., \& Damkier, P. (2014). Alcohol and breastfeeding. Basic \& Clinical Pharmacology \& Toxicology, 114(2), 168-173 [Review].

Hekmatpanah, J., Haghighat, N., \& Adams, C. R. (1994). Alcohol consumption by nursing rats and its effect on the cerebellum of the offspring. Alcohol and Alcoholism, 29(5), 535-547

Ho, E., Collantes, A., Kapur, B. M., Moretti, M., \& Koren, G. (2001). Alcohol and breast feeding: calculation of time to zero level in milk. Biology of the Neonate, 80(3), 219-222

Huang, L., Wang, Y., Zhang, L., Zheng, Z., Zhu, T., Qu, Y., et al. (2018). Maternal smoking and attention-deficit/hyperactivity disorder in offspring: A meta-analysis. Pediatrics. https://doi.org/10. 1542/peds.2017-2465

Joseph, R. M., Korzeniewski, S. J., Allred, E. N., O'Shea, T. M., Heeren, T., Frazier, J. A., et al. (2017). Extremely low gestational age and very low birthweight for gestational age are risk factors for autism spectrum disorder in a large cohort study of 10-yearold children born at 23-27 weeks' gestation. American Journal of Obstetrics and Gynecology, 216(3), 304.e301-304.e316. https:// doi.org/10.1016/j.ajog.2016.11.1009

Jung, Y., Lee, A. M., McKee, S. A., \& Picciotto, M. R. (2017). Maternal smoking and autism spectrum disorder: meta-analysis with population smoking metrics as moderators. Scientific Reports, 7(1), 4315. https://doi.org/10.1038/s41598-017-04413-1

Kesäniemi, Y. A. (1974). Ethanol and acetaldehyde in the milk and peripheral blood of lactating women after ethanol administration. BJOGl An International Journal of Obstetrics \& Gynaecology, 81(1), 84-86. https://doi.org/10.1111/j.1471-0528.1974.tb00369.x

Kim, J. U., Majid, A., Judge, R., Crook, P., Nathwani, R., Selvapatt, N., et al. (2020). Effect of COVID-19 lockdown on alcohol consumption in patients with pre-existing alcohol use disorder. Lancet Gastroenterol Hepatol, 5(10), 886-887. https://doi.org/10.1016/ s2468-1253(20)30251-x

Lawton, M. E. (1985). Alcohol in breast milk. Australian \& New Zealand Journal of Obstetrics \& Gynaecology, 25(1), 71-73

Lebel, C., Roussotte, F., \& Sowell, E. R. (2011). Imaging the impact of prenatal alcohol exposure on the structure of the developing human brain. Neuropsychology Review, 21(2), 102-118. https:// doi.org/10.1007/s11065-011-9163-0

Leigh, A., \& Gong, X. (2010). Does maternal age affect children's test scores? Australian Economic Review, 43(1), 12-27. https://doi. org/10.1111/j.1467-8462.2009.00573.x

Luck, W., \& Nau, H. (1984). Nicotine and cotinine concentrations in serum and milk of nursing smokers. British Journal of Clinical Pharmacology, 18(1), 9-15

Mennella, J. (2001). Alcohol's effect on lactation. Alcohol Research and Health, 25(3), 230-234

Mennella, J. A., Pepino, M. Y., \& Teff, K. L. (2005). Acute alcohol consumption disrupts the hormonal milieu of lactating women. Journal of Clinical Endocrinology \& Metabolism, 90(4), 1979-1985

Museridze, D. P., Tsaishvili Ts, S., Svanidze, I. K., \& Khanayeva, Z. (2008). Disorders in memory and learning in offspring of alcoholized female rats, and a possibility for correction of these changes. Neurophysiology, 40(2), 115-120
Napierala, M., Mazela, J., Merritt, T. A., \& Florek, E. (2016). Tobacco smoking and breastfeeding: Effect on the lactation process, breast milk composition and infant development. A Critical Review. Environmental Research, 151, 321-338

Neill, E., Meyer, D., Toh, W. L., van Rheenen, T. E., Phillipou, A., Tan, E. J., et al. (2020). Alcohol use in Australia during the early days of the COVID-19 pandemic: Initial results from the COLLATE project. Psychiatry and Clinical Neurosciences. https://doi.org/ 10.1111/pcn.13099

Noland, J. S., Singer, L. T., Short, E. J., Minnes, S., Arendt, R. E., Lester Kirchner, H., et al. (2005). Prenatal drug exposure and selective attention in preschoolers. Neurotoxicology and Teratology, 27(3), 429-438. https://doi.org/10.1016/j.ntt.2005.02.001

Panagiotidis, P., Rantis, K., Holeva, V., Parlapani, E., \& Diakogiannis, I. (2020). Changes in alcohol use habits in the general population, during the COVID-19 lockdown in greece. Alcohol and Alcoholism (Oxford, Oxfordshire), 55(6), 702-704. https://doi.org/10. 1093/alcalc/agaa092

Paolozza, A., Rasmussen, C., Pei, J., Hanlon-Dearman, A., Nikkel, S. M., Andrew, G., et al. (2014). Deficits in response inhibition correlate with oculomotor control in children with fetal alcohol spectrum disorder and prenatal alcohol exposure. Behavioural Brain Research, 259, 97-105. https://doi.org/10.1016/j.bbr.2013.10.040

Rosen, B., Lee, B., Lee, N., Yang, Y., \& Burstyn, I. (2015). Maternal smoking and autism spectrum disorder: A meta-analysis. Journal of Autism and Developmental Disorders, 45(6), 1689-1698. https://doi.org/10.1007/s10803-014-2327-z

Semrud-Clikeman, M. (2005). Neuropsychological aspects for evaluating learning disabilities. Journal of Learning Disabilities, 38(6), $563-568$

Singer, A. B., Aylsworth, A. S., Cordero, C., Croen, L. A., DiGuiseppi, C., Fallin, M. D., et al. (2017). Prenatal alcohol exposure in relation to autism spectrum disorder: Findings from the study to explore early development (SEED). [Research Support, U.S. Gov't, P.H.S. Research Support, N.I.H., Extramural]. Paediatric and Perinatal Epidemiology, 31(6), 573-582

Soloff, C., Lawrence, D., \& Johnstone, R. (2005). LSAC technical paper no. 1: Sample design. Melbourne: Australian Institute of Family Studies.

Sterne, J. A., White, I. R., Carlin, J. B., Spratt, M., Royston, P., Kenward, M. G., et al. (2009). Multiple imputation for missing data in epidemiological and clinical research: Potential and pitfalls. BMJ, 338, b2393

Stoet, G., \& Geary, D. C. (2013). Sex differences in mathematics and reading achievement are inversely related: Within- and acrossnation assessment of 10 years of PISA data. PLOS ONE, 8(3), e57988

Tang, S., Wang, Y., Gong, X., \& Wang, G. (2015). A meta-analysis of maternal smoking during pregnancy and autism spectrum disorder risk in offspring. International Journal of Environmental Research and Public Health, 12(9), 10418-10431. https://doi.org/ 10.3390/ijerph120910418

Tong, S., Baghurst, P., Vimpani, G., \& McMichael, A. (2007). Socioeconomic position, maternal IQ, home environment, and cognitive development. The Journal of Pediatrics, 151(3), 284-288.e281

Van Heugten, C. M., Hendriksen, J., Rasquin, S., Dijcks, B., Jaeken, D., \& Vles, J. H. S. (2006). Long-term neuropsychological performance in a cohort of children and adolescents after severe paediatric traumatic brain injury. Brain Injury, 20(9), 895-903

Verhaeghen, P., \& Salthouse, T. A. (1997). Meta-analyses of age-cognition relations in adulthood: estimates of linear and nonlinear age effects and structural models. Psychological Bulletin, 122(3), 231-249 
von Hippel, P. T. (2016). The number of imputations should increase quadratically with the fraction of missing information. arXiv preprint arXiv 1608.05406.

Wetherill, L., Foroud, T., \& Goodlett, C. (2018). Meta-analyses of externalizing disorders: genetics or prenatal alcohol Exposure? Alcoholism: Clinical \& Experimental Research, 42(1), 162-172

Wilens, T. E., Martelon, M., Joshi, G., Bateman, C., Fried, R., Petty, C., et al. (2011). Does ADHD predict substance-use disorders? A 10-year follow-up study of young adults with ADHD. Journal of the American Academy of Child \& Adolescent Psychiatry, 50(6), 543-553. https://doi.org/10.1016/j.jaac.2011.01.021

Zhu, J. L., Olsen, J., Liew, Z., Li, J., Niclasen, J., \& Obel, C. (2014). Parental smoking during pregnancy and ADHD in children: The Danish national birth cohort. Pediatrics, 134(2), e382-388
Zhu, J., Takita, M., Konishi, Y., Sudo, M., \& Muramatsu, I. (1996). Chronic nicotine treatment delays the developmental increase in brain muscarinic receptors in rat neonate. Brain Research, 732(1-2), 257-260

Publisher's Note Springer Nature remains neutral with regard to jurisdictional claims in published maps and institutional affiliations. 